\title{
HIGHER TAXA IN THE FOSSIL RECORD AND THE RECOGNITION OF EVOLUTIONARY EVENTS: PATTERNS VS. PROCESSES
}

FASTOVSKY*, David E., Dept. of Geology, Univ. of Rhode Island, Kingston, RI 02881-0807, U.S.A.; SHEEHAN, Peter M., Dept. of Geology, Milwaukee Public Museum, 800 W. Wells St., Milwaukee, WI 53233, U.S.A.

The fossil record is commonly (but not universally) invoked as an historical record with which to test evolutionary scenarios. The process by which this takes place involves the recognition of patterns of taxa in a temporal framework. The patterns are in turn interpreted as the result of some evolutionary mechanism. We submit that if the fossil record is to be a tool in evolutionary studies, the use of appropriately-defined higher taxa can be consistent with the objective of recognizing patterns from which evolutionary events can be inferred.

We implemented a study to monitor the ecological diversity of "families" of dinosaurs through the last 2.2 million years of the Cretaceous in the terrestrial sediments of the Hell Creek Formation in eastern Montana. We concluded, using the Shannon index and rarefaction as monitors, that at the "family" level, the ecological diversity of dinosaurs remained constant.

We suspect that the taxa we designated as "families" did not branch ancestrally at equivalent points in their respective phylogenies. Nevertheless, each is a monophyletic group, established and united by synapomorphies. We therefore measured and tracked the relative proportions of monophyletic groups through sequential time slices of the latest Cretaceous. We interpreted this as a measure of ecological diversity.

The phylogenetic methods that were used to define the extinct "families" in our study are epistemologically indistinguishable from methods that are used to establish monophyletic fossil groups at any taxonomic level, including the lowest levels. Why should special significance be attributed to a taxonomic level (i.e., species) that is obtained by means no different from those used to obtain the other levels?

Use of the fossil record in evolutionary reconstruction requires the recognition of patterns, and the attribution of evolutionary mechanisms to those patterns. It is unlikely that selection acts on units other than populations which arguably, are not even taxa. Recognition of historical patterns rather than causality or process, therefore, may be the unique province of the stratigraphic record. 